H I G H L I G H T S

\section{IN BRIEF}

EVOLUTION

\section{Prevalence of small inversions in yeast gene order evolution.}

Seoighe, C. et al. Proc. Natl Acad. Sci. USA 97, 14433-14437 (2000)

A comparison of the genome sequences of two eukaryotes, Saccharomyces cerevisiae and Candida albicans, reveals that small inversions have been a strong force during the evolution of gene order. Only $9 \%$ of the genes that are adjacent in one yeast are also adjacent in the other and, of these, 35\% correspond to genes that are adjacent but differ in orientation or order. The authors propose that neighbourhoods of conserved genes characterized by altered orientation or order have arisen through successive small inversions that involve regions containing fewer than ten genes.

\section{MULTIFACTORIAL GENETICS}

\section{Familial aggregation of Parkinson's disease in Iceland.} Sveinbjörnsdóttir, S. et al. N. Engl. J. Med. 343, 1765-1770 (2000)

Parkinson disease was thought to be a multifactorial trait caused by the interaction between multiple genes and the environment. However, recent research questions whether genetic factors contribute to the late-onset form of the disease, which affects individuals over 50 years of age. Genetic studies on the lateonset form of the disease have been limited, so Sveinbjörnsdóttir et al. used the Icelandic population-based genealogical record to determine whether patients were more likely to be related than control subjects. Their results clearly show that late-onset Parkinson disease does have a genetic component in the Icelandic population.

\section{COMPARATIVE GENOMICS}

\section{Zebrafish comparative genomics and the origins of vertebrate chromosomes.}

Postlethwait, J. H. et al. Genome Res. 10, 1890-1902 (2000)

\section{A comparative map of the zebrafish genome. Woods, I. G. et al. Genome Res. 10, 1903-1914 (2000)}

Two collaborative papers from the labs of John Postlethwait and Will Talbot provide new zebrafish genome mapping data and a comparative analysis of zebrafish and human gene maps that shed light on vertebrate evolution. The groups mapped 691 previously unlocalized zebrafish genes and ESTs, and from these and previously mapped loci, they identified 804 putative human orthologues. A subsequent comparison of human and zebrafish gene maps revealed 139 new conserved syntenies and the lack of a one-to-one correspondence between human and zebrafish chromosomes, possibly because of a genome duplication event in an early zebrafish ancestor. Despite this, human and zebrafish genomes have roughly the same number of chromosomes, perhaps because of an excess of chromosome fissions in the tetrapod lineage.

\title{
FUNCTIONAL GENOMICS
}

\section{It's a bug's life}

Caulobacter crescentus is the kind of bacterium it's a pleasure to have around. This single-celled, aquatic organism (which belongs to the same subclass as the less friendly Rickettsia and Agrobacterium) is the most prevalent non-pathogenic bacterium in nutrient-poor freshwater streams, and is one of the organisms responsible for sewage treatment and for removing heavy metals from waste water. Its properties make it interesting to study from a developmental point of view. For example, at each cell division, $C$. crescentus divides into two morphologically distinct daughter cells (shown in the photo): one that resembles the mother (that is, a stalked cell) and a free-swimming cell (a swarmer) that bears a flagellum. C. crescentus is also an ideal system in which to do transcriptional studies. Unlike multicellular organisms, in which gene expression is coordinated by external cues and hormonal signals, the important steps in C. crescentus's life cycle depend entirely on the internal environment, that is, on the relationship between its genes.

Now that the genomic sequence of C. crescentus's single $4-\mathrm{Mb}$ chromosome is complete, post-genomic efforts are underway to understand how the expression profiles of this bug's genes relate to each other. A recent report in Science has shown that the transcription of $19 \%$ of $C$. crescentus's genes (of which there are nearly 3,000 ) is temporally controlled, and among these are genes that regulate the cell cycle.

Laub and colleagues found that the expression profiles of 553 genes varied in a cyclical pattern during the cell cycle, peaking at discrete times. Among these were genes that had previously been shown to have cellcycle-related functions. For many of these genes, expression rose just before or coincident with the cellcycle-related event itself: for example, genes required for nucleotide synthesis were switched on just before the onset of DNA replication.
Furthermore, genes whose products were part of a multi-protein complex — the whip-like flagellum, for example - were co-expressed.

The cell cycle can run smoothly only if the timing of each event is precisely coordinated with that of others - an order that is easiest to achieve in the presence of a regulatory hierarchy. CtrA is a critical response regulator whose activity depends on the cell cycle and may be at the top of one such hierarchy. The study showed that the expression of $26 \%$ of cell-cycleregulated genes was affected by a lossof-function mutation in CtrA. Over a quarter of these genes had promoters with the consensus CtrA binding sites, and are therefore good candidates for direct regulation by CtrA.

This leaves 409 genes still outstanding whose expression is cellcycle-dependent but that are not influenced by CtrA. Further studies should focus on identifying which master regulatory proteins - there are 27 candidates - account for the periodic expression of these genes.

Tanita Casci

(2) References and links ORIGINAL RESEARCH PAPER Laub, M. T. et al. Global analysis of the genetic network controlling a bacterial cell cycle. Science $\mathbf{2 9 0}$ 2144-2148 (2000)

WEB SITES Lucy Shapiro's lab | Experimental data

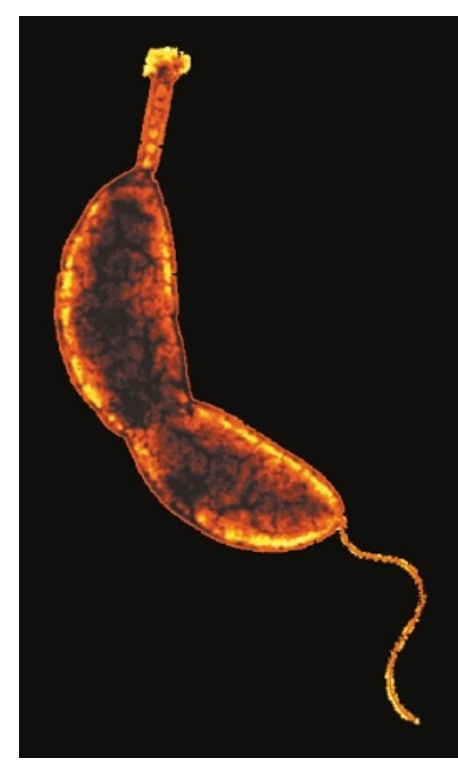

Courtesy of Yves Brun. 